\title{
XXXVII. An investigation of the properties of lactic acid
}

\author{
Jacob Berzelius M.R.A.
}

To cite this article: Jacob Berzelius M.R.A. (1813) XXXVII. An investigation of the properties of lactic acid , Philosophical Magazine Series 1, 41:180, 241-247, DOI: $10.1080 / 14786441308638740$

To link to this article: http://dx.doi.org/10.1080/14786441308638740

里 Published online: 27 Jul 2009.

Submit your article to this journal $₫$

Џ Article views: 2

Q View related articles $\square$ 


\section{[ 2A1]}

XXXVII. An Investigation of the Properties of Lactic Acid. By J JCOB Berzelius, Professor of Medicine and Pharmacy, and M.R.A. Stockholm. Translated from the Swedish*.

$\mathrm{T}$ sour milk to coagulate, filtered the fluid, and boiled it away to $18 \mathrm{th}$; he then filtered it again, to separate the curd which was deposited. This acid fluid he saturated with lime water, which threw down a precipitate of bone earth; the remaining lime was cautiously separated by the addition of oxalic acid, taking care that it should not be in excess, and the fluid was evaporated to dryness. The acid was then dissolved in alcohol, which, after evaporation, afforded it in the purest state that was then known. Scheele determined the relations of this acid to the greatest number of bases so clearly and accurately, that there was no reason to entertain any doubt of its proper nature, as essentially distinct from all otber acids. The assertions of Scheele have however within the last few years not only been called in question, but even positivcly contradicted, by some French chemists, Bouillon Lagrange, Thenard, Fourcroy, and Vauquelin. Since only the two last chemists bave had sufficient confidence in their scientific character to decide positively on this question, in contradiction to the resuits of Scheele's experiments, I shall here only say a few words respecting their assertions. Among the properties of the lactic acid, $S$ cheele has mentioned that it is not volatile, and that it is destroyed by distillation p $_{\text {, }}$ and affords a sour spirit; a name, by which the empyreumatic vinegar, which is obtained in the distillation of organic substances, was then always distinguished. In their investigations respecting this acid, the French chemists saturated it with an alkali, mixed the salt with concentrated sulphuric acid, and distilled the mixture. The liguid distilled contained a weak vinegar, which was saturated with alkali, was evaporated to dryness, and was distilled again with sulphuric acid. It requires no great depth of chemical knowledge to discover the impropriety of this mode of operation, and the necessity of the formation of vinegar at the expense of the component parts of the lactic acid destroyed. On the other hand, these chemists seem not to have paid the slightest attenvion to the proper characteristic salts, which are produced by the lactic acid with different bases, and from

* From the Animal Chemistry of Professor Berzelius, vol. ii. p. 480.

Vol. 41. No.180. April 1813. $\mathrm{Q}$ which 


\section{An Investigation of the Properties of Lactic Acid.}

which we usually derive the principal and the most certain criterions of an acid. They concluded from their experiments, that Scheele had been mistaken, and that the lactic acid $w$ as nothing else than a combination of the acetic with a proper animal matter, which hindered the volatilisation of the acid.

Since we have found that the lactic acid acts a more or less distinguished part in the blood, in muscular flesh, in the marrow, in urine, and in milk, it appears desirable that these circumstances should be very accurately examined. 1 have therefore undertaken to investigate its nature very fully, and have obtained, by means of a long and often repeated series of different experiments, a complete conviction that Scheele was in the right, and that the lactic acid is a peculiar acid, very distinct from all others.

I shall here relate the results of my experiments. The extract which is obtained when dried whey is digested with alcohol, contains, as I have already observed, uncombined lactic acid, lactate of potass, muriate of potass, and a proper animal matter. I mixed this solution in alcohol with another portion of alcohol to which $\frac{1}{75}$ of concentrated sulphuric acid tad been added, and continued to add fresh portions of this mixture as long as any saline precipitate was formed, and until the fluid had acquired a decidedly acid taste. Some sulphate of potass was precipitated, and there remained in the alcohol muriatic acid, lactic acid, sulphuric acid, and a minute portion of phosphoric acid, detached from some bone earth which harl been held in solution. The acid liquor was filterce, and afterwards digested with carbonate of lead, which with the lactic acid affords a salt soluble in alcohol. As soon as the mixture had acquired a sweetish taste, the three mineral acids bad fallen down in combination with the lead, and the lactic acid remained behind, imperfectly saturated by a portion of it, from which it was detached by means of sulphuretted bydrogen, and then evaporated to the consistence of a thick varnish, of a dark-brown colour and sharp acid taste, but altogether without smell.

In order to free it from the animal matter which might remain combined with it, I boiled it with a mixture of a large quantity of fresh lime and water, so that the animal substances were precipitated and destroyed by the lime. The lime became yellow brown, and the solution almost coluurless, while the mass emitted a smell of soap lees, which disappeared as the boiling was continued. The fluid thus obtained was filtered and evaporated, until a great part 
of the superfluous lime held in solution was precipitated. A small portion of it was then decomposed by oxalic acid, and carbonate of silver was dissolved in the uncombined lactic acid, until it was fully saturated. With the assistance of the lactate of silver thus obtained, a further quantity of muriatic acid was separated from the lactate of lime, which was then decomposed by pure oxalic acid, free from nitric acid, taking care to leave it in such a state that neither the oxalic acid nor lime water afforded a precipitate. It was then evaporated to dryness, and dissolved again in alcohol, a small portion of oxalate of lime, before retained in union with the acid, now remaining undissolved. The alcohol was evaporated until the mass was no longer fluid while warm; it became a brown clear transparent acid, which was the lactic acid, free from all substances that we have hitherto had reason to think likely to contaminate it.

The lactic acid, thus purified, has a brown yellow colour, and a sharp sour taste, which is much weakened by diluting it with water. It is without smell in the cold, but emits, when heated, a sharp sour smell, not unlike that of sublimed oxalic acid. It cannot be made to crystallize, and does not exhibit the slightest appearance of a saline substance, but dries into a thick and smooth varnish, which slowly attracts moisture from the air. It is very easily soluble in alcohol. Heated in a gold spoon over the flame of a candle, it first boils, and then its pungent acid smell becomes very manifest, but extremely distinct from that of the acetic acid; afterwards it is charred, and has an empyreumatic, but by no means an animal smell. $\mathbf{A}$ porous charcoal is left behind, which does not readily burn to ashes. When distilled, it gives an empyreumatic oil, water, empyreumatic vinegar, carbonic acid, and inflammable gases. With alkalis, eartbs, and metallic oxides, it affords peculiar salts; and these are distinguished by being soluble in alcohol, and in general by not having the least disposition to crystallize, but drying into a mass like gum, which slowly becomes moist in the air.

Laclate of polass is obtaincd, when the lactate of lime, purified as has been mentioned, is mixed warm with a warm solution of carbonate of potass. It forms in drying a gummy, light yellow brown, transparent mass, which cannot easily be made hard. If it is mixed with concen. trated sulphuric acid, no sinell of acetic acid is perceived; but if the mixture is heated, it acquires a disagreeable pungent snell, which is observable in all animal substances mixed 


\section{In Investigation of the Properties of Lactic Act.}

with the sulphuric acid. The extract, which is obtained directly from milk, contains this salt; but this affords, when mixed with sulphuric acid, a sharp acid smell, not unlike that of the acetic acid. This however depends not on acetic but on muriatic acid, which in its concentrated state introduces this modification into the smell of almost all organic bodies. The pure lactate of potass is easily snluble in alcohol; that which contains an excess of potass, or is still contaminated with the animal matter soluble in alcohol, which is destroyed by the treat ment with line, is slowly soluble, and requires about 4 parts of warm alcohol for its solution. It is dissolved in boiling alcohol more abundantly than in cold, and separates from it, white it is cooling, in the form of bard drops.

The lactate of sota resembles that of potass, and can only be distinguished from it by analysis.

Lactate of ammionia. If concentrated lactic acid is sa. turated with caustic ammonia in excess, the mixture acquires a strong volatile smell, not unlike that of the acefate or formiate of ammonia, which however soon ceases. The salt which is left has sometimes a slight tendency to shoot into crystals. It affords a gummy mass, which in the air acquires an excess of acidily. When heated, a great part of the alkali is expelled, and a very acid salt remains, which deliquesces in the air.

The lactate of laryta may he obtained in the same way as that of lime; but it then contains an excess of the base. When evaporated, it affords a gummy mass, soluble in alcohol. A portion remains undissolved, which is a subsalt, is doughy, and has a browner colour. That which is dissolved in the alcohol affords by evaporation an almost colourless gummy mass, which hardens into a stiff but not a brittle varnish. It does not show the least tendency to crystallize. The salt which is less soluble in alcobol may be further purified from the animal matter adbering to it, by adding to it more bary!a, and then becomes more soluble.

The lactate of lime is obtained in the manner above described. It affords a gummy mass, which is also divided by alcohol into two portions. The larger portion is soluble, and gives a shining varnish inclining to a light yellow colour, which, when slowly dried, cracks all over, and becomes opake. This is pure lactate of lime. That which is insoluble in alcohol is a powder, with excess of the base; received on a filter, it becomes smooth in the air like gam, 
ar like malate of lime. By boiling with more lime, and by the precipitation of the superfinous base upon exposure to the air, it becomes pure and soluble in alcohol.

Lactate of magnesia, evaporated to the consistence of a thin syrup, and left in a warm place, shoots into small granular crystals. When hastily evaporated to dryness, it affords a gummy mass. With regard to alcohol, its properties resemble those of the iwo preceding salts.

Ammoniaco-nagnesian lactate is obtained by mixing the preceding salt with caustic ammonia, as long as any precipitation continues. Liy spontaneous evaporation this salt shoots into needle-shaped prisms, which are little coloured, and do not change in the air. I have once seen these crystals form in the alcoholic extract of milk boiled to dryness: but this is by no means a common occurrence.

The lactate of silver is procured by dissolving the carbonate in the lactic acid. The solution is of a light yellow somewhat inclining to green, and has an unpleasant taste of verdigris. When evaporated in a flat vessel, it dries into a very transparent greenish yellow varnish, which has externally an unusual splendour like that of a looking-glass. If the evaporation is conducted in a deeper vessel, and with a stronger heat, a part of the salt is decomposed, and remains brown from the reduction of the silver. If this salt is dissolved in water, no inconsiderable portion of the silver is reduced and deposited, even when the salt has been trausparent; and the concentrated solution has a fine greenish yellow colour, which by dilution becomes yellow. If we dissolve the oxide of silver in an impure acid, the salt becomes brown, and more silver is revived during the evaporation.

The lactate of the protoxide of mercury is obtained when the lactic acid is saturated with black oxidated mercury. It has a light yellow colour, which disappears by means of repeated solution and evaporation. The salt exhibits acid properties, deliquesces in the air, and is partially dissolved in alcohol, but is at the same time decomposed, and deposits carbonate of mercury, while the mixture acquires a slight smell of ether. The lactic acid dissolves also the yed oxide of mercury, and gives with it a red gummy deli. quescent salt. If it is lett exposed to a warm and moist atmosphere, it deposits, after the expiration of some weeks, a light semi-crystalline powder, which I have not examined, but which probably must be acetate of mercury.

The lactate of lead may be obtained in several different Q 3 dcgrees 


\section{An Investigation of the Properties of Lactic Acid.}

degrees of saturation. If the lactic acid is digested with the carbonate of lead, it becomes browner than before, but cannot be fully saturated with the oxide; and we obtain an acid salt, which does not crystallize, hut dries into a syruplike brown mass, with a sweet austere taste. When a soJution of lacuc acid in alcohol is digested with finely powdered litharge, until the solution becnmes sweet, and is then slowly evaporated to the consistence of honey, the neutral lactate of lead crvstallizes in swall gravish grains, which may be rinsed with alcohol, to wash off the viscid mass that adheres to them, ind will then appear as a gray granular salt, which when dry is light and silvery, like the precipitate thrown down by alcohol from a precipitated alkali. It is not changed in the air; treated with sulphuretted hydrogen, it aff ords pare lactic acid. If the lactic acid is digested with a greater portion of levigated litharge than is required for its saturation, the fluid acquires first a hrowner colour, and as the digestion is continued, the colour becomes more and more pale, and the oxide swell's into a bulky powder, of a colour somewhat lighter than before. If the fluid is evaporated, and water is then poured on the dry mass, a very small portion of it only is dissolved; the solution is not coloured, and when it is exposed to the air, a pellicle of carbonate of lead is separated from it. If the dried salt of lead be boiled with water, and the solution be filtered while hot, a great part of that which had been dissolved will be precipitated while it cools, in the form of a white or light yellow powder, which is a sublactate of lead, This salt is of a light flame colour; when dried, it remains mealy, and soft to the touch, and it is decomposed by the weakest acids, while the acid salt is dissolved in water, exhibiting a sweet taste and a brown colour. When moistened with water, it undergoes this change from the operation of the carbonic acid diffused in the air. If this salt is warmed and then set on fire at one point, it burns like tin. der, and leaves the lead in great measure reduced. A hundred parts of this salt, dissolved in nitric acid, and precipitated with carbonate of potass, gave exactly 100 parts of carhonate of lead; consequently its component parts, determined trom those of the carbonate, must be 83 of the oxide of leacl, and 17 of the lactic acid. At the same time we cannot wholly depend on this proportion, and it certainly makes the quantity of lead somewhat ton great. The relation of the lactic acid to lead affords one of the best methods of secognising it, and I have always principally employed 
employed it, in extracting this acid from animal fluids; it gives the clearest distinction between the lactic acid and the acetic.

The lactate of iron is of a red brown colour, does not erystallize, and is not soluble in alcohol. The lactate of zinc crystallizes. Both these metals are dissolved by the lactic acid, with an exirication of hydrngen gas. The lactate of copper, according to its different degrees of saturation, waries from blue to green and dark blue. It does not erystallize.

It is vily necessary to compare the descriptions of these salts with what we know of the salts which are formed with the same bases by other acids, for example, the acetic, the malic, and others, in order to be completely convinced that the lactic acid must be a peculiar acid, pertectly distinct from all others.

XXXVIII.Critical Observations on Dr. WoLLAsTon's stated Improvement of the Camera Olscura and Microscope in the Application of the Meniscus and two Plano-convex Lenses; proving their Inferiority to the doulle Convex Lens gentrally used. By WiLlia m Jones, Optician.

\section{To Mr. Tilloch.}

SIR,- $I_{N}$ your impartial Journal, vol. xvii. and also in another cotemporary Journal *, some observations of mine were published, proving satisfacturily, I trust, that the periscopic spectacle glass advertised by Dr. Wollaston as possessing a new optical principle, and affording an improvement in the figure of a spectacle glass, wa. no other than the old rejected neviscus lens; contained no refractive property different from the plano-convex and double convex lenses; but, as it caused a greater degree of aberration than those two lenses, was a worse form of lens for spectacles or any other instrument than the double convex lens generally used by practical opticians;it must, therefore, surprise others conversant in optics, besides myself, that Dr. Wollaston should be induced again to propose the meniscus in the camera obscura instead of the double convex lens; his account of which is copied into yoir journal of last month from the Philosophical Transactions for 1812 .

The desire that I have to maintain an optical truth, and the duty $I$ owe to our professional interests, oblige me to

- Nicholson's Journal, vol, vii. 